\title{
Insights into the improved macrolide inhibitory activity from the high-resolution cryo-EM structure of dirithromycin bound to the $E$. coli $70 S$ ribosome
}

\author{
EVGENY B. PICHKUR, ${ }^{1,2,12}$ ALENA PALESKAVA, ${ }^{1,3,12}$ ANDREY G. TERESHCHENKOV, ${ }^{4}$ PAVEL KASATSKY, ${ }^{1}$ \\ EKATERINA S. KOMAROVA, ${ }^{5,6}$ DMITRII I. SHIRIAEV ${ }^{4}$ ALEXEY A. BOGDANOV, ${ }^{4}$ OLGA A. DONTSOVA ${ }^{4,6,7}$ \\ ILYA A. OSTERMAN, ${ }^{4,6}$ PETR V. SERGIEV, ${ }^{4,6}$ YURY S. POLIKANOV, ${ }^{8,9}$ ALEXANDER G. MYASNIKOV, ${ }^{1,10,11}$ \\ and ANDREY L. KONEVEGA ${ }^{1,2,3}$ \\ ${ }^{1}$ Petersburg Nuclear Physics Institute named by B.P. Konstantinov of NRC "Kurchatov Institute," Gatchina, 188300, Russia \\ ${ }^{2}$ National Research Center "Kurchatov Institute," Moscow, 123182, Russia \\ ${ }^{3}$ Peter the Great St. Petersburg Polytechnic University, Saint Petersburg, 195251, Russia \\ ${ }^{4}$ Department of Chemistry and A.N. Belozersky Institute of Physico-Chemical Biology, Lomonosov Moscow State University, \\ Moscow, 119992, Russia \\ ${ }^{5}$ Department of Bioengineering and Bioinformatics and A.N. Belozersky Institute of Physico-Chemical Biology, Lomonosov Moscow State \\ University, Moscow, 119992, Russia \\ ${ }^{6}$ Skolkovo Institute of Science and Technology, Skolkovo, Moscow region, 143025, Russia \\ ${ }^{7}$ Shemyakin-Ovchinnikov Institute of Bioorganic Chemistry, Russian Academy of Sciences, Moscow, 117997, Russia \\ ${ }^{8}$ Departments of Biological Sciences and Pharmaceutical Sciences, University of Illinois at Chicago, Chicago, Illinois 60607, USA \\ ${ }^{9}$ Center for Biomolecular Sciences, University of Illinois at Chicago, Chicago, Illinois 60607, USA \\ ${ }^{10}$ Department of Structural Biology, St. Jude Children's Research Hospital, Memphis, Tennessee 38105, USA \\ ${ }^{11}$ Centre for Integrative Biology, IGBMC, CNRS, Inserm, Université de Strasbourg, Illkirch, 67404, France
}

\begin{abstract}
Macrolides are one of the most successful and widely used classes of antibacterials, which kill or stop the growth of pathogenic bacteria by binding near the active site of the ribosome and interfering with protein synthesis. Dirithromycin is a derivative of the prototype macrolide erythromycin with additional hydrophobic side chain. In our recent study, we have discovered that the side chain of dirithromycin forms lone pair- $\pi$ stacking interaction with the aromatic imidazole ring of the His69 residue in ribosomal protein uL4 of the Thermus thermophilus 70S ribosome. In the current work, we found that neither the presence of the side chain, nor the additional contact with the ribosome, improve the binding affinity of dirithromycin to the ribosome. Nevertheless, we found that dirithromycin is a more potent inhibitor of in vitro protein synthesis in comparison with its parent compound, erythromycin. Using high-resolution cryo-electron microscopy, we determined the structure of the dirithromycin bound to the translating Escherichia coli 705 ribosome, which suggests that the better inhibitory properties of the drug could be rationalized by the side chain of dirithromycin pointing into the lumen of the nascent peptide exit tunnel, where it can interfere with the normal passage of the growing polypeptide chain.
\end{abstract}

Keywords: dirithromycin; macrolide; antibiotic; 70S ribosome; cryo-EM; inhibitor; nascent peptide exit tunnel

\section{INTRODUCTION}

High therapeutic efficacy and safety ensured that erythromycin (ERY; Fig. 1A, right), a macrolide antibiotic, and its semisynthetic analogs had joined aspirin and penicillin as

\footnotetext{
${ }^{12}$ These authors contributed equally to this work.

Corresponding authors: yuryp@uic.edu, alexander.myasnikov@ stjude.org, konevega_al@pnpi.nrcki.ru

Article is online at http://www.rnajournal.org/cgi/doi/10.1261/rna. 073817.119.
}

the most widely used drugs in the history of medicine (Ying and Tang 2010). Various chemical modifications of ERY resulted in several macrolides with improved antibacterial activities and pharmacological properties. One of 


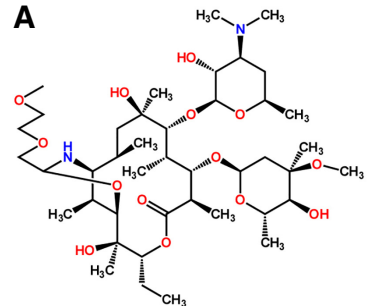

DIRITHROMYCIN (DIR)

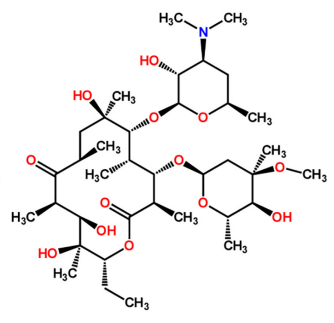

ERYTHROMYCIN (ERY)
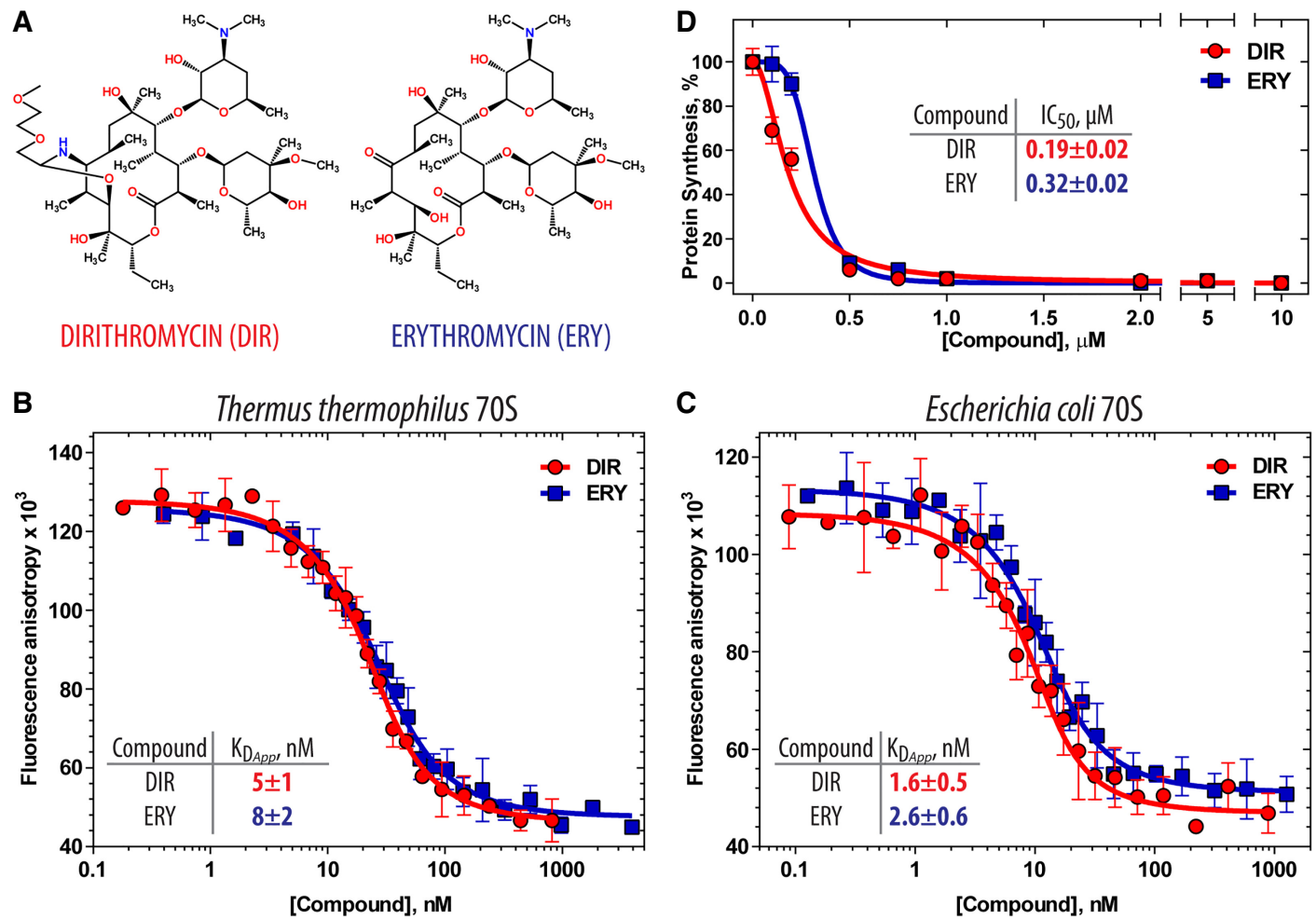

FIGURE 1. Binding and inhibition properties of dirithromycin and erythromycin. (A) Chemical structures of macrolides dirithromycin (DIR, left) and erythromycin (ERY, right). ( $B, C)$ Competition-binding assay to test the displacement of fluorescently labeled BODIPY-ERY analog from the T. thermophilus $(B)$ or $E$. coli $(C) 70$ S ribosomes in the presence of increasing concentrations of DIR, or ERY measured by fluorescence anisotropy. All the reactions were repeated four times. Error bars represent standard deviation. The resulting values for apparent dissociation constants $\left(K_{D a p p}\right)$ are shown on each plot. (D) Inhibition of protein synthesis by increasing concentrations of DIR or ERY in the in vitro cell-free transcription-translation coupled system. The relative enzymatic activity of in vitro synthesized firefly luciferase is shown. Error bars represent standard deviations of the mean of three independent measurements.

the second-generation macrolides, dirithromycin (DIR, Fig. $1 \mathrm{~A}$, left), differs from the parent ERY by the presence of a 6membered oxazine ring and a hydrophobic (2-methoxyethoxy)-methyl side chain that has significantly increased the delivery of this antibiotic to tissues due to its higher lipophilicity (Counter et al. 1991). Although DIR has been used for years in the clinic to treat uncomplicated bacterial infections of the respiratory tract, skin, and soft tissues (Brogden and Peters 1994), the interaction of DIR with its primary target-bacterial ribosome-remains poorly explored.

Based on its chemical structure, it is highly unlikely that DIR would interact with the $70 \mathrm{~S}$ ribosome in a manner that is principally different from other macrolides: resting of the macrolactone ring on the surface formed by the nucleobases of residues A2058, A2059, and C2611 of the 23S rRNA at the entrance to the nascent peptide exit tunnel (NPET) near the peptidyl transferase center (PTC) (Bulkley et al. 2010; Dunkle et al. 2010; Svetlov et al. 2019). Structural features of the side chains of macrolides define their individual properties and the mechanism of inhibition depending on the degree of obstruction of the NPET as well as on their ability to directly or allosterically affect the activity of the PTC (Poulsen et al. 2000; Tenson et al. 2003; Kannan et al. 2014).

A recent structural work by Khabibullina et al. revealed that the hydrophobic side chain of DIR encroaches upon the previously unexplored binding pocket within the NPET of the Thermus thermophilus (Tth) $70 S$ ribosome and forms a contact with the ribosomal protein uL4. This additional contact might be responsible for an overall stronger binding of DIR to the ribosome compared with ERY that lacks the tail. However, the part of the ribosomal protein uL4 that specifically interacts with the DIR side chain is different in various bacteria making this contact species-specific. The same DIR side chain could either interact differently with the protein $\mathrm{UL} 4$ in the ribosomes from other bacteria, such as Escherichia coli (Eco) or do not interact with uL4 at all. Another possibility is that instead of anchoring the drug on the ribosome, the side chain of DIR could reside in the lumen of NPET and simply block the passage of the nascent polypeptide chain through it. This leaves an open question of whether the side chain of DIR could have any function(s) associated with the pharmacological target of the drug, the ribosome. 
In this work, we report the results of biochemical studies that suggest that DIR exhibits similar binding affinity both to the Tth and Eco 70 S ribosomes compared to its parent compound ERY. At the same time, DIR demonstrates more pronounced protein synthesis inhibitory properties in comparison with ERY. We have also obtained a high-resolution cryo-electron microscopy (cryo-EM) structure of DIR bound to the translating Eco 70 S ribosome, which, better than the thermophilic analog, resembles the ribosomes of medically important pathogens. The high resolution (2.1 A) and excellent quality of the obtained charge density map, especially around the DIR binding site, allowed us to visualize the ribosome-bound drug molecule and its interactions with the ribosome. Interestingly, unlike the recent structure of Tth $70 \mathrm{~S}$ ribosome in complex with DIR, we do not observe any additional contacts between the side chain of DIR and the $70 S$ ribosome from Eco, suggesting that the side chain plays a little-to-no role in the anchoring of the drug on the ribosome. The observed orientation of the DIR side chain into the lumen of NPET suggests that it likely interferes with the passage of the nascent polypeptide chain, rationalizing better inhibitory properties of DIR versus ERY.

\section{RESULTS AND DISCUSSION}

\section{Dirithromycin and erythromycin bind to the $70 \mathrm{~S}$ ribosomes from different bacteria with similar affinities}

In our recent structural study of DIR bound to the 705 ribosome from thermophilic bacterium Thermus thermophilus (Tth) we found that the hydrophobic (2-methoxyethoxy)-methyl side chain of DIR forms lone pair- $\pi$ stacking interaction with the aromatic imidazole ring of the His69 residue in the ribosomal protein uL4 (Khabibullina et al. 2019). This additional contact with the ribosome could potentially endow DIR with better binding properties compared to the macrolides not having such chemical moiety (for example, ERY). To check this hypothesis, we used a competition-binding assay exploiting BODIPY-labeled erythromycin (BODIPY-ERY), which allows measuring the affinities of macrolides and their derivatives for the ribosome (Yan et al. 2005; Tereshchenkov et al. 2016, 2018). We expected that all of the studied macrolide compounds would show equal or similar affinities compared to BODIPY-ERY. The apparent dissociation constant ( $\left.K_{\text {Dapp }}\right)$ of BODIPY-ERY $(43 \pm 2$ $\mathrm{nM}$ ) was determined by its direct equilibrium binding to the Tth 70S ribosomes (Supplemental Fig. S3). The $K_{\text {Dapp }} \mathrm{S}$ of DIR and ERY, obtained using competition with BODIPY-ERY ( $5 \pm 1 \mathrm{nM}$ and $8 \pm 2 \mathrm{nM}$, respectively), indicated that DIR and ERY bind to the Tth ribosome with similar affinities (Fig. 1B). This result suggests that even if the interaction of the (2-methoxyethoxy)-methyl side chain of DIR contributes to the drug anchoring in the $T$ th $70 \mathrm{~S}$ ribosome, this effect is relatively minor.

By aligning the sequences of ribosomal protein uL4 we have previously discovered that the same contact of DIR side chain with the wall of NPET would be impossible in the $70 \mathrm{~S}$ ribosomes from other bacterial species because the residue equivalent to His69 of the Tth protein uL4, which contacts DIR in Tth ribosome, is non-conserved in other bacteria (Supplemental Fig. S4; Khabibullina et al. 2019). Unlike T. thermophilus and mycoplasmas that contain histidine, the majority of bacteria (including E. coli) have glycine residue (Gly64 in E. coli) at this position, and, therefore, cannot be involved in the same type of lone pair- $\pi$ stacking interactions with the ribosome-bound DIR molecule. Nevertheless, in our previous work, we speculated that in the absence of uL4 histidine residue, the side chain of DIR could establish an alternative stabilizing contact with one of the adjacent residues in the ribosome (for example, Arg61 in the loop of ribosomal protein uL4 in Eco 70 S ribosome [Khabibullina et al. 2019]). To test this hypothesis and to finally determine whether the DIR side chain is important for binding to the ribosomes of various species or not, we measured the affinities of DIR and ERY for Eco $70 S$ ribosome. The apparent dissociation constant $K_{\text {Dapp }}$ of DIR and ERY determined by competition with BODIPY-ERY was $1.6 \pm 0.5 \mathrm{nM}$ and $2.6 \pm 0.6 \mathrm{nM}$, respectively (Fig. 1C). The observed value of $K_{\text {Dapp }}$ for ERY is consistent with the values ranging from 2 to $36 \mathrm{nM}$ determined previously by different groups using Eco 705 ribosomes (Goldman et al. 1994; Hansen et al. 1999; Dinos et al. 2003; Yan et al. 2005; Tereshchenkov et al. 2016; Svetlov et al. 2017). Similar to the results obtained for the Tth ribosomes, these data indicate that DIR has an affinity for the Eco $70 S$ ribosomes, which is statistically indistinguishable from that of ERY. Nevertheless, because both drugs demonstrate somewhat stronger binding to the medically relevant $70 \mathrm{~S}$ ribosomes from Eco in comparison to the ones from Tth, we decided to continue our studies with the Eco 70 S ribosomes.

\section{Dirithromycin is a potent inhibitor of protein synthesis}

To gain further insight into DIR inhibitory activity, we assessed its ability to interfere with the in vitro protein synthesis. We compared the inhibitory effects of DIR and the parent compound ERY on the translation of a firefly luciferase reporter in the E. coli S30 extract. DIR demonstrated nearly twofold better inhibition compared to ERY $\left(I C_{50}=\right.$ $0.19 \pm 0.02$, and $0.32 \pm 0.02 \mu \mathrm{M}$, respectively) (Fig. 1D).

Since the DIR molecule possesses a hydrophobic side chain attached to a rigid 6-membered oxazine ring, neither of which the ERY molecule has, these data suggest that the overall better ribosome inhibitory properties of DIR could be due to the presence of these chemical moieties. 
Apparently, the observed stronger inhibitory properties of DIR on the Eco $70 S$ ribosomes cannot be rationalized by the same contact with the protein uL4 as in the Tth 70S ribosome because the same contact in the Eco $70 \mathrm{~S}$ is impossible. However, there is still a possibility that the side chain of DIR forms a yet unknown additional contact with the ribosome or simply points into the open space of the NPET where it could interfere with the normal passage of a nascent polypeptide chain. To address this possibility, we set to determine a cryo-EM structure of the Eco $70 S$ ribosome in complex with DIR.

\section{Dirithromycin exhibits canonical macrolide binding to the $70 \mathrm{~S}$ ribosome}

To determine the mode of binding of DIR to the actively translating Eco 705 ribosome, we obtained a highresolution cryo-EM structure of DIR bound to the functional mRNA-programmed ribosomal complex, containing deacylated $\operatorname{tRNA}_{i}^{\text {Met }}$ in the $P$ site and formed in situ dipeptidyl fMet-Phe-tRNA $^{\text {Phe }}$ in the A site. Focused refinement of the $50 \mathrm{~S}$ ribosomal subunit yielded a $2.1 \AA$ cryoEM density map, however, the local resolution of the obtained map was not isotropic. Many of the core areas of the $50 S$ subunit exhibited high-resolution features such as water molecules, $\mathrm{Mg}^{2+}$ ions and even "holes" visible in the aromatic purine/pyrimidine rings and ribose (Supplemental Fig. S1C). Subsequent 3D classification of the obtained data set resulted in separate classes of complexes: one class containing densities for both the A- and the P-site tRNA substrates, and the other class containing density only for the P-site tRNA. A comparison of these two classes allowed us to visualize conformational changes in the $23 \mathrm{~S}$ rRNA upon binding and accommodation of the A-site tRNA.

As expected, DIR binds to the Eco $70 \mathrm{~S}$ ribosome in the canonical macrolide binding pocket, which is located in the upper part of the NPET in the heart of the large ribosomal subunit. As in the case with other macrolides, DIR binding to the ribosome is stabi- lized by a hydrogen bond ( $\mathrm{H}$-bond) between the $2^{\prime}-\mathrm{OH}$ group of the desosamine sugar of the drug and the N1 atom of the nucleotide A2058 of the 23S rRNA. All other electrostatic interactions appeared to be relatively weak with the distances between the atoms being more than 3.5 A. Fitting of the DIR chemical structure (Fig. 1A) into the obtained cryo-EM charge density map reveals an almost complete match (Fig. 2A,B). The only exception is
A

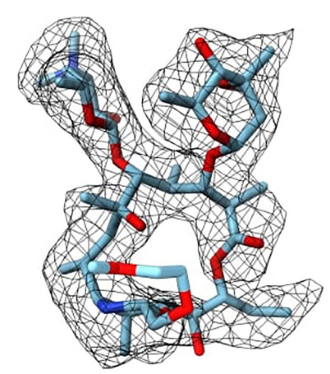

C

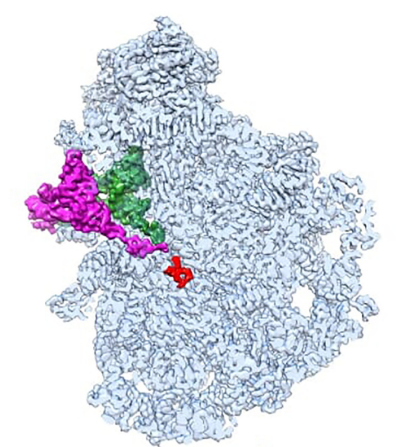

E

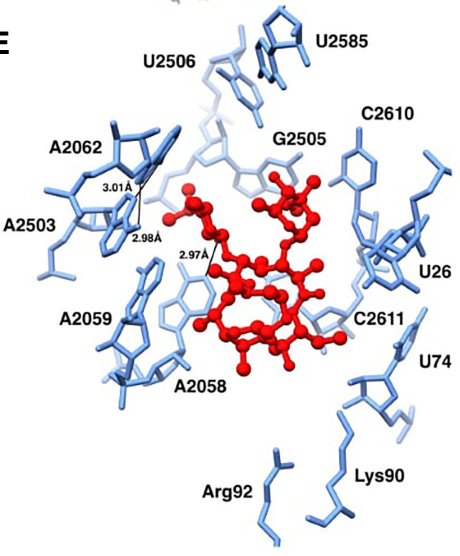

B

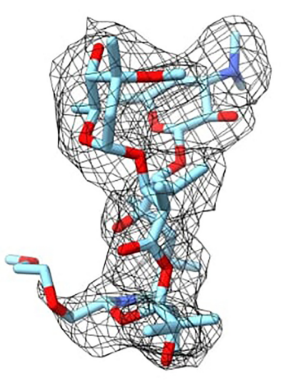

D

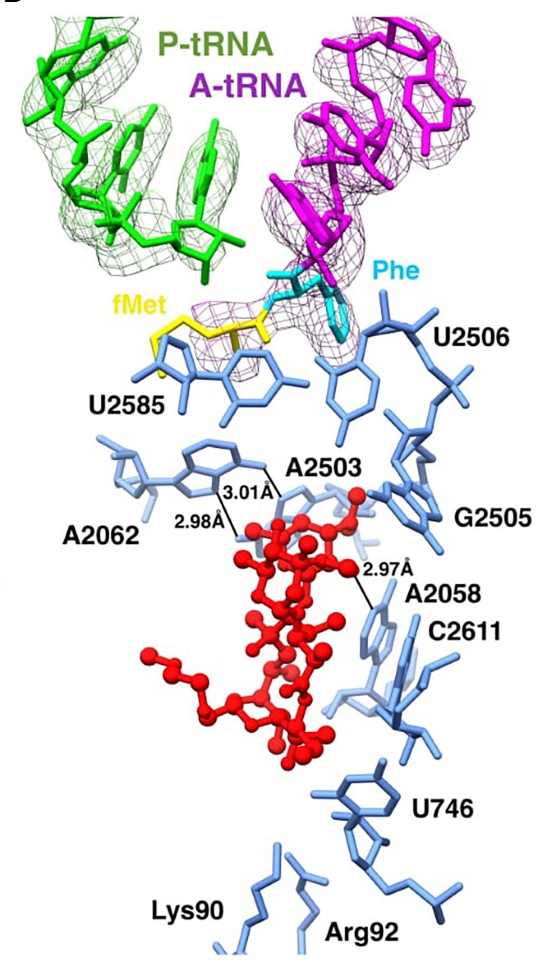

FIGURE 2. The structure of antibiotic DIR in complex with the $E$. coli $70 S$ ribosome. $(A, B)$ Coulomb potential map of DIR in complex with the E. coli ribosome (black mesh). The fitted model of the compound is displayed in its respective charge density viewed from two different perspectives. Carbon atoms are colored light blue, nitrogen atoms are blue, oxygen atoms are red. (C) Overview of the DIR binding site on the E. coli large ribosomal subunit (light blue) viewed from the cytoplasm onto the A site. DIR is shown in red, A-site tRNA and P-site tRNA are shown in magenta and green, respectively. The $30 S$ subunit is not shown. $(D, E)$ Close-up views of the DIR binding site in the ribosome exit tunnel. E. coli numbering of the nucleotides in the $23 \mathrm{~S}$ rRNA is used. Potential $\mathrm{H}$-bond interactions are indicated with black lines. Note that the nucleotide A2062 of the 23S rRNA forms a symmetric trans A-A Hoogsteen/Hoogsteen base pair with the residue $m^{2} A 2503$. Panel $D$ also shows the experimentally observed charge density map for the A-site fMet-Phe dipeptidyl-tRNA ${ }^{\text {Phe }}$ (magenta) and the P-site deacylated tRNA ${ }_{i}^{\text {Met }}$ (green). 
the distal part of the (2-methoxyethoxy)-methyl side chain of DIR, some parts of which lack corresponding charge density, pointing to the flexibility of this part of the molecule. Unlike in the recent X-ray crystal structure of DIR bound to the Tth 70S ribosome (Khabibullina et al. 2019), the hydrophobic tail of DIR in our new structure resides in the open space of the NPET and is located far from the walls of the tunnel (Fig. 2C-E; Supplemental Movie S1). At the same time, based on our biochemical data (Fig. 1B,C), the lack of additional contact does not lead to a decrease in the binding affinity of DIR to the Eco 70 S ribosome compared to the Tth one, suggesting the prevailing importance of the standard interaction with the nucleotide A2058 of the 23S rRNA for binding of this macrolide to the ribosome. Therefore, the enhanced inhibitory activity of DIR is likely to be associated with its side chain pointing directly into the open space of the NPET (Fig. 2D), where it could partially obstruct the tunnel and interfere with the passage of the growing polypeptide chain. Although we have a dipeptide in our structure, it is too short to reach the location of the DIR side chain and, thus, our speculation could not be verified directly at this moment.

Detailed analysis of vicinity of the macrolide binding site in our structure allowed us to visualize a macrolide-induced structural rearrangement, which has been observed in the numerous previous structures of the macrolide-bound $T$ th $70 S$ ribosomes, but not in the Eco 705 ribosomes-the rotation of the nucleotide A2062 of the 23S rRNA by $\sim 160^{\circ}$ into a position where it forms a symmetric trans AA Hoogsteen/Hoogsteen base pair with the residue $\mathrm{m}^{2} \mathrm{~A} 2503$ (Fig. 3A; Bulkley et al. 2010; Almutairi et al. 2017; Khabibullina et al. 2019; Svetlov et al. 2019). Interestingly, the classical macrolide ERY does not cause the same reorientation of the nucleotide A2062 in the vacant (Dunkle et al. 2010) or stalled (Arenz et al. 2016) ribosomes from
E. coli (Fig. 3B, turquoise), Staphylococcus aureus (Fig. 3B, blue; Halfon et al. 2019), as well as in the large ribosomal subunits from eubacterium Dienococcus radiodurans (Schlünzen et al. 2001) or archaeon Haloarcula marismortui (Fig. 3B, yellow; Tu et al. 2005). Our cryo-EM structure revealed that the desosamine sugar of DIR induces the same structural rearrangement of A2062 (Fig. 3B) that was previously observed only in the macrolide-bearing Tth $70 \mathrm{~S}$ ribosomes (Fig. 3A). Therefore, we suggest that, unlike Tth $70 \mathrm{~S}$ ribosome, in which macrolide binding appears to be sufficient for the A2062 reorientation to take place, the same structural transformation in the Eco $70 \mathrm{~S}$ ribosome does not occur simply as a result of macrolide binding and requires a yet unknown additional signal. One possibility is that the functionally active elongation ribosome complex, which we have used here for the structure determination, can produce such a signal.

An important aspect of macrolide action is the allosteric predisposition of the PTC to translation arrest (VazquezLaslop et al. 2008; Arenz et al. 2014; Sothiselvam et al. 2014). For example, data on chemical modification as well as all-atom molecular dynamics simulations suggested that binding of ERY to vacant ribosome results in a significant reorientation of the nucleotide $\mathrm{U} 2585$ in the $23 \mathrm{~S}$ rRNA located more than $8 \AA$ away from the macrolide binding pocket (Sothiselvam et al. 2014). The effect of ERY binding on the position of nucleotide U2585 has also been observed previously in the cryo-EM structure of the ErmBL-stalled nascent chain complex (Arenz et al. 2016). At the same time, the orientation of the $\mathrm{U} 2585$ relative to the tRNA substrates is essential for the peptidyl transferase reaction to occur efficiently (Schmeing et al. 2005; Polikanov et al. 2014b). In the drug-free H. marismortui $50 \mathrm{~S}$ ribosomal subunit with the vacant A site, the U2585 nucleotide shields the labile ester bond of the peptidyl-
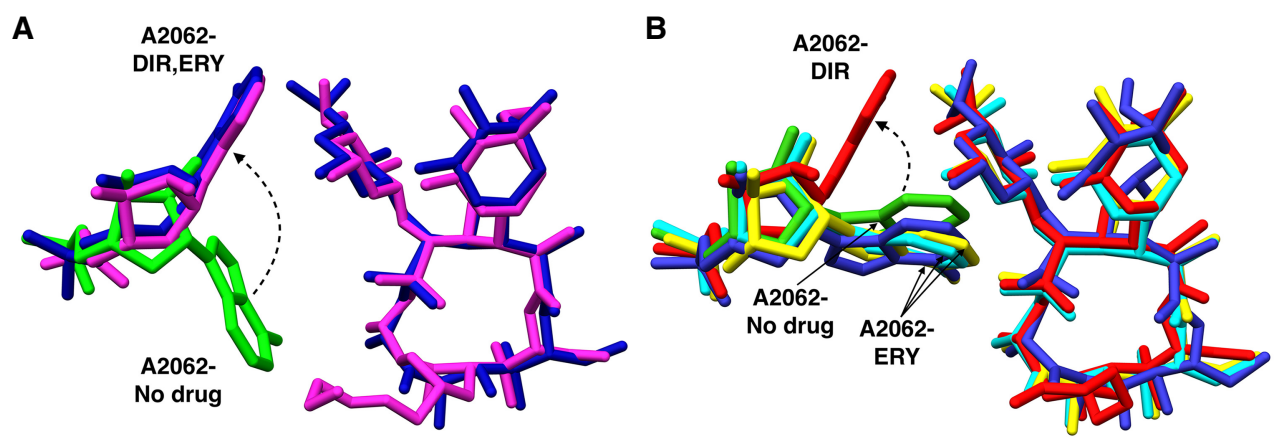

FIGURE 3. DIR-induced structural rearrangement of the A2062 nucleotide. (A) Rotation of the nucleotide A2062 of the $23 \mathrm{~S}$ rRNA by 160 degrees in the Tth 70S ribosome upon binding of the ERY (dark blue, PDB entry 4V7X [Bulkley et al. 2010]) or DIR (magenta, PDB entry 6OF1 [Khabibullina et al. 2019]). The structure of the drug-free Tth 70S ribosome (green) is taken from the PDB entry1VY5 (Polikanov et al. $2014 b)$. (B) Positions of the nucleotide A2062 of the 23S rRNA in the current DIR-ribosome structure (red) compared to its positions in the previous structures of the ribosome from different species in complex with ERY: Haloarcula marismortui 50S (yellow, PDB entry 1YI2 [Tu et al. 2005]); Escherichia coli 70S (cyan, PDB entry 4V7U [Dunkle et al. 2010]); Staphylococcus aureus 70S (blue, PDB entry 6S0Z [Halfon et al. 2019]). The structure of the drug-free Eco 70 S ribosome (green) is taken from the PDB entry 4YBB (Noeske et al. 2015). All structures were aligned based on domain $V$ of the $23 \mathrm{~S}$ rRNA. 

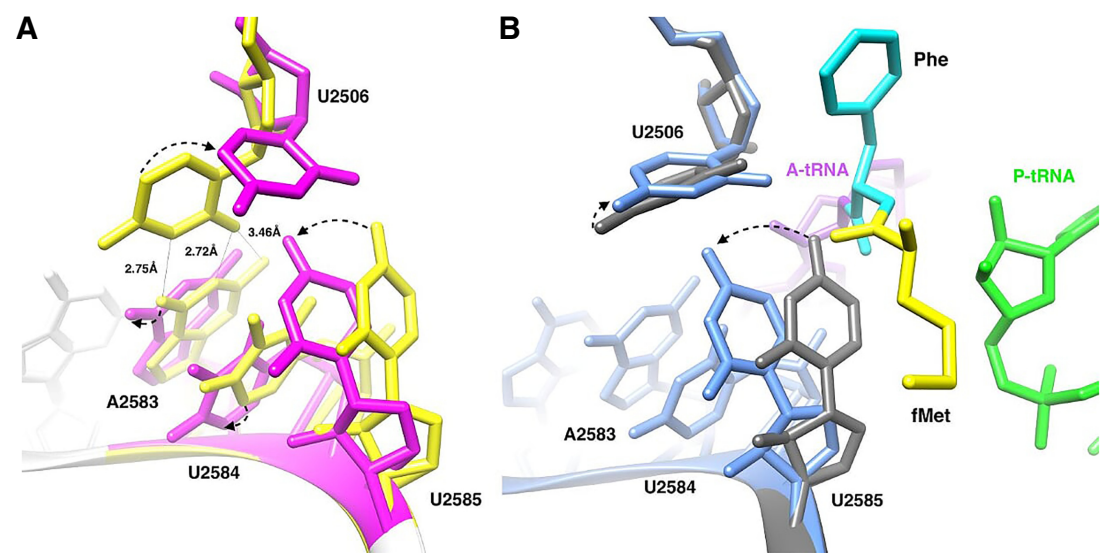

FIGURE 4. Conformational changes in the $23 \mathrm{~S}$ rRNA resulting from accommodation of the Asite substrate. (A) Comparison of the $H$. marismortui $50 \mathrm{~S}$ structures in the absence (yellow, uninduced state, PDB entry 1VQ6 [Schmeing et al. 2005]) and presence (magenta, induced state, PDB entry 1VQN [Schmeing et al. 2005]) of the A-site substrate. Note that binding of the A-site tRNA-mimic results in rotation of the nucleotide U2506 from a location where it forms a wobble base pair with the nucleotide G2583 (uninduced state of the PTC) to a location where it forms one side of the A-site cleft (induced state of the PTC). (B) Comparison of the structures without (gray) and with (blue) the A-site-bound full-length tRNA obtained in the current work. All structures were aligned based on domain $\mathrm{V}$ of the $23 \mathrm{~S}$ rRNA.

tRNA in order to prevent its hydrolysis and premature release of the nascent peptide (Schmeing et al. 2005). The same nucleotide reorients upon accommodation of an aminoacyl-tRNA into the A site and allows peptidyl transfer to occur (Fig. 4A; Schmeing et al. 2005). Thus, any changes in the position of the U2585 caused by the macrolide binding could lead to impaired functioning of the PTC. Therefore, in addition to the antibiotic binding site, we have carefully analyzed whether any of the key functional nucleotides around the PTC undergo DIR-induced structural rearrangements. To assess the potential dynamics of the PTC nucleotides in the presence of DIR we have compared cryo-EM maps of the two ribosome complexes that we obtained in this study: (i) the main population of the complexes with A- and P-site tRNA substrates (fMetPhe-tRNA ${ }^{\text {Phe }}$ in the $A$ site and deacylated tRNA ${ }_{i}^{\text {Met }}$ in the $P$ site, respectively) and (ii) a subpopulation of the complexes containing only the unreacted P-site tRNA (fMettRNA ${ }_{i}{ }^{\text {Met }}$ ). Our analysis shows that binding of DIR in the context of functional $70 \mathrm{~S}$ ribosome complex does not alter the position of the U2585 nucleotide (Fig. 4B), which remains the same as in the drug-free ribosome (Fig. 4A). Minor shifts of U2583 and U2584 that were previously observed upon accommodation of the A-site tRNA substrate (Schmeing et al. 2005) could not be unambiguously identified in the unsharpened cryo-EM map. Another key nucleotide in the PTC, U2506, retains an "open" conformation regardless of the presence of the A-site substrate that was previously shown for Tth 705 ribosomes (Polikanov et al. 2014b). Nucleotide A2602, which plays a crucial role in the proper positioning of the tRNA CCAends in the PTC, is distinctly visible in our map underlining similar locations of the tRNA molecules in DIR-bound and antibioticfree ribosome complexes. Altogether, our data suggest that binding of DIR does not cause any significant rearrangements of the key nucleotides in the PTC, at least in the case of Eco $70 \mathrm{~S}$ ribosome at the initiation or pretranslocation stages.

In summary, our functional and structural studies of dirithromycin revealed it as a more potent inhibitor of in vitro protein synthesis in comparison with its parent compound, erythromycin. This effect could be attributed to the side chain of DIR pointing into the lumen of the NPET, where it can interfere with the normal passage of the growing polypeptide chain. The present work examines the structural features of the macrolide binding pocket and the PTC of the functional ribosome complex from E. coli containing the in situ synthesized dipeptide attached to the tRNA in the A site upon binding of the macrolide antibiotic DIR. The obtained data reveal no significant structural rearrangements as the short peptide does not protrude to the macrolide binding site and does not bear a stalling sequence. However, a very high resolution of our cryo-EM density map provides the basis for further investigation of ribosome complexes containing longer non-stalling nascent peptides immersing to different depths into the NPET. Structural data obtained from such complexes will help us uncover the details of the molecular mechanism of context-specific inhibition by macrolide antibiotics.

\section{MATERIALS AND METHODS}

\section{Materials for biochemical experiments}

Dirithromycin (DIR) and erythromycin (ERY) were provided by Victor G. Kartsev from Interbioscreen Ltd.

\section{In vitro binding assay}

Binding affinities (apparent dissociation constants) of tested macrolides to the E. coli or T. thermophilus $70 S$ ribosomes were analyzed by a competition-binding assay using fluorescently labeled erythromycin, BODIPY-ERY, as described before (Yan et al. 2005; Tereshchenkov et al. 2016, 2018). Briefly, BODIPY-ERY (4 nM) was incubated with the ribosomes ( $12 \mathrm{nM}$ for Eco and $24 \mathrm{nM}$ for Tth) for $30 \mathrm{~min}$ at $25^{\circ} \mathrm{C}$ in buffer containing $20 \mathrm{mM}$ HEPES-KOH $(\mathrm{pH}$ 7.5), $50 \mathrm{mM} \mathrm{NH}_{4} \mathrm{Cl}, 10 \mathrm{mM} \mathrm{Mg}\left(\mathrm{CH}_{3} \mathrm{COO}\right)_{2}, 4 \mathrm{mM} \beta$-mercaptoethanol, and $0.05 \%(\mathrm{v} / \mathrm{v})$ Tween-20. The solution of a tested 
compound in a range of concentrations from $0.05 \mathrm{nM}$ to $1 \mu \mathrm{M}$ was added to the formed complex. The mixture was incubated for 30 min and the values of fluorescence anisotropy were measured using Victor X5 multilabel plate reader (PerkinElmer).

\section{In vitro translation analysis}

Inhibition of firefly luciferase synthesis by DIR, or ERY was assessed essentially as described previously (Polikanov et al. 2014a). Briefly, the in vitro T7-transcribed firefly luciferase mRNA was translated in the E. coli S30 cell-free system prepared according to (Svetlov et al. 2006). Reactions programmed with $200 \mathrm{ng}$ mRNA were carried out in $5 \mu \mathrm{L}$ aliquots at $37^{\circ} \mathrm{C}$ for 30 $\mathrm{min}$, and the activity of in vitro synthesized luciferase was assessed using $5 \mu \mathrm{L}$ of the substrate from the Steady-Glo Luciferase Assay System (Promega) on Victor X5 multilabel plate reader (PerkinElmer).

\section{S complex preparation for cryo-EM}

Ribosomes, EF-Tu, and initiation factors from E. coli were prepared as described previously (Milon et al. 2007). Met-tRNA $_{i}{ }^{\text {Met }}$ and Phe-tRNA ${ }^{\text {Phe }}$ were purified by HPLC (Milon et al. 2007). The mRNA was a 92-nt-long derivative of $\mathrm{m022}$ mRNA with the coding sequence AUGUUU. Initiation complex (70S/mRNA/fMet-

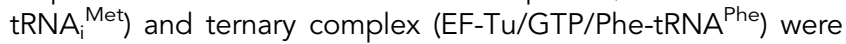
prepared as described previously (Konevega et al. 2007). The pretranslocation complex with deacylated tRNA ${ }_{i}{ }^{\text {Met }}$ in the $P$ site and dipeptidyl $\mathrm{fMet}^{-P h e-t R N A^{\mathrm{Phe}}}$ in the A site was prepared by mixing initiation and ternary complexes in buffer containing $50 \mathrm{mM}$ HEPES-KOH (pH 7.5), $70 \mathrm{mM} \mathrm{NH}_{4} \mathrm{Cl}, 30 \mathrm{mM} \mathrm{KCl}, 7 \mathrm{mM} \mathrm{MgCl}_{2}$ at $37^{\circ} \mathrm{C}$ (Konevega et al. 2007). The complex was stabilized by increasing the concentration of $\mathrm{MgCl}_{2}$ to $21 \mathrm{mM}$ and purified by gel filtration on a Biosuite 450 HR 5- $\mu$ m column (Waters). Finally, $70 \mathrm{~S}$ ribosome complex $(0.3 \mu \mathrm{M})$ was incubated with DIR $(30 \mu \mathrm{M})$ and spermine $(0.6 \mathrm{mM})$ at $0^{\circ} \mathrm{C}$ for $5 \mathrm{~min}$ before application onto $\mathrm{EM}$ grids. Quantifoil R2/2 grids coated with an additional $10 \mathrm{~nm}$ amorphous carbon film were glow-discharged for $45 \mathrm{sec}$ at 25 $\mathrm{mA}$ using PELCO easiGlow. A total of $2.5 \mu \mathrm{L}$ of the sample was applied onto the grids and plunge-frozen in liquid ethane using Vitrobot Mark IV (Thermo Fisher) at $10^{\circ} \mathrm{C}$ and $100 \%$ humidity (Khatter et al. 2015).

\section{Cryo-EM data collection and processing}

Cryo-EM data were collected using Cs-corrected Titan Krios (Thermo Fisher) transmission electron microscope, equipped with a Falcon II direct electron detector. Cs corrector was tuned every $12 \mathrm{~h}$ in order to avoid aberrations. Movies were recorded at defocus values from -0.3 to $-2.2 \mu \mathrm{m}$ at a magnification of $75,000 \times$, which corresponds to the pixel size of $0.86 \AA$ at the specimen level. During 1.4-sec exposure 28 frames were collected with the total dose of $\approx 80 \mathrm{e}^{-} / \AA^{2}$, while the first and the last frames were discarded. Motion correction was performed using MotionCor2 algorithm (Zheng et al. 2017) implemented in the Relion 3.0 software (Zivanov et al. 2018). CTF parameters were determined using GCTF (Zhang 2016) and refined later in Relion 3.0 and CisTEM (Grant et al. 2018). Particles were picked with
Gautomatch (https://www.mrc-lmb.cam.ac.uk/kzhang/) using low-pass filtered templates. Two rounds of the 2D classification using $8 \times$ binned particles (pixel size $=6.88 \AA$ A $/$ pixel) were performed to eliminate ice, carbon edges, and false-positive particles containing noise resulting in approximately 549,000 70S particles for further analysis. 3D refinement followed by a 3D classification was performed to separate classes with dissociated $50 \mathrm{~S}$ subunits and low-quality particles using $4 \times$ binned particle (pixel size $=3.44 \AA$ A $/$ pixel) images. A total of 401,905 particles were selected for a high-resolution refinement without binning in Relion 3.0 followed by CTF refinement with a mask covering $50 S$ subunit. Particle polishing was performed with the following parameters estimated from the data set: $\sigma_{V}=0.86 \AA /\left(\mathrm{e}^{-} / \AA^{2}\right), \sigma_{D}=$ $10,485 \AA, \sigma_{A}=0.78 \AA /\left(e^{-} / \AA^{2}\right)$ followed by another round of $3 D$ refinement, CTF refinement and particle polishing (Zivanov et al. 2019). Particles without binning (512-pixel box, $0.86 \AA ̊$ /pixel) were exported to CisTEM. 3D auto-refine was performed in CisTEM followed by few more rounds of the manual refinement with a soft mask covering $50 S$ subunit. Resolution based on the $\mathrm{FSC}=0.143$ criteria after refining with a $50 \mathrm{~S}$ mask was $2.24 \AA$ (Supplemental Fig. S1A). Additional rounds of manual refinement were performed with the mask covering the $A$ and $P$ sites as well as a $40 \AA$ spherical part of the 50 S subunit near the DIR binding site. This density map was used for the primary analysis of the DIR interactions with the ribosome as well as for the model building of the A-site MMet-Phe-tRNA $^{\text {Phe }}$ and the deacylated P-site tRNA $_{i}{ }^{\text {Met }}$. Focused 3D classification of the 70S data set into five classes with a spherical mask covering the $A$ and $P$ sites was performed in CisTEM to separate a class with the strong density for Aand P-site substrates and a class containing only the P-site tRNA (Supplemental Fig. S2). The other three classes were not included in the analysis as they demonstrated either a weak density for the substrate or an incomplete separation across classes. The first class (with both A- and P-site tRNAs present) contained 117,919 particles ( $30 \%$ of the total number of particles), while the second class contained 65,269 particles ( $16 \%$ of particles). Resolution of the density maps was estimated at $2.54 \AA$ and $2.66 \AA$, respectively (Supplemental Table S2). Finally, high-order aberrations were corrected using RELION 3.1 (beta) (https://www.biorxiv.org/content/ $10.1101 / 798066 \mathrm{v} 1$ ) to reconstruct a higher resolution structure of the $50 \mathrm{~S}$ subunit. Another 3D refinement resulted in a $2.1 \AA$ structure of the $50 \mathrm{~S}$ subunit (Supplemental Fig. S1A), while some areas at the center of the map show high-resolution features corresponding to the local resolution below the global estimation (Supplemental Fig. S1B). The final map after the correction of high-order aberrations was used to build a final model of the $50 \mathrm{~S}$ subunit. The density maps sharpened in CisTEM or Relion were used to produce figures.

\section{Model building}

We used a high-resolution X-ray structure of the E. coli $70 S$ ribosome (PDB entry 4YBB [Noeske et al. 2015]) as a starting model. Structures of the A-site $f M$ et-Phe-tRNA ${ }^{\text {Phe }}$ and the P-site deacylated tRNA ${ }_{i}^{\text {Met }}$ were taken from the PDB entry $1 \mathrm{VY} 5$ (Polikanov et al. 2014b) and docked into the charge density map using Coot (Emsley and Cowtan 2004). Because in our experiments we used tRNA ${ }^{\text {Phe }}$ from yeast, while the A-site tRNA ${ }^{\text {Phe }}$ in the PDB entry $1 \mathrm{VY} 5$ was from $E$. coli, we have changed all the 
nucleotides in the starting model to obtain the sequence of yeast tRNA $^{\text {Phe }}$. The atomic model of DIR was generated from its known chemical structure (Fig. 1A) using PRODRG online software (Schüttelkopf and van Aalten 2004), which was also used to generate restraints for energy minimization and refinement based on idealized 3D geometry. Atomic model and restraints were used to $\mathrm{fit} /$ refine DIR into the obtained charge density map. Additionally, we have removed ribosomal protein L9 from the model due to the lack of density in the observed cryo-EM map. Also, ribosomal proteins L10 and L11, part of the 23S rRNA (A1042-A1113), and part of the 23S rRNA (A2096-2193) corresponding to the L1 stalk were removed from the model before the structure refinement. The final model of the $50 \mathrm{~S}$ ribosomal subunit in complex with DIR and tRNAs was generated by multiple rounds of model building in COOT, followed by refinement in PHENIX (Adams et al. 2010). Removed parts of the model were rigid-body fitted into the map to preserve geometry and added to the final model. The models of the $70 S$ ribosome in complex with DIR and with and without the dipeptidyl fMet-Phe-tRNA ${ }^{\text {Phe }}$ were prepared by the rigid-body fitting of the final $50 S$ structure with tRNAs and the $30 \mathrm{~S}$ structure from PDB entry 4 YBB. The statistics of data collection and refinement are compiled in Supplemental Table S1. All figures showing atomic models were generated using PyMol (www.pymol.org), Chimera (Pettersen et al. 2004) and ChimeraX (Goddard et al. 2018) software.

\section{DATA DEPOSITON}

Coordinates and cryo-EM density maps were deposited in the RCSB Protein Data Bank with accession code PDB6XZ7 and Electron Microscopy Database with accession code EMD-10655 for the Eco 50S ribosomal subunit in complex with dirithromycin, A-, and P-site tRNAs; PDB6XZA and EMD-10656 for the E. coli $70 S$ ribosome in complex with dirithromycin, mRNA, and deacylated P-site tRNA $_{i}{ }^{\text {Met }}$ (focused classification); PDB-6XZB and EMD10657 for the $E$. coli $70 S$ ribosome in complex with dirithromycin, mRNA, A-site dipeptidyl fMet-Phe-tRNA ${ }^{\text {Phe }}$ and deacylated P-site tRNA ${ }_{i}^{\text {Met }}$ (focused classification).

\section{SUPPLEMENTAL MATERIAL}

Supplemental material is available for this article.

\section{ACKNOWLEDGMENTS}

We thank Dr. Victor G. Kartsev for providing dirithromycin. We thank all members of the Y.S.P., A.A.B., P.V.S., and A.L.K. laboratories for discussions and critical feedback. We thank Dr. Maksim S. Svetlov and Egor A. Syroegin for critical reading of the manuscript and valuable suggestions. This work was supported by the Russian Science Foundation [grant 17-14-01416 to A.L.K. (cryo-EM experiments), grant 18-44-04005 to I.A.O. (biochemical characterization of antibiotic's interaction with the ribosome)], Illinois State startup funds [to Y.S.P.], National Institutes of Health [grants R01-GM132302 and R21-Al137584 to Y.S.P.], the Russian Foundation for Basic Research [grant 17-00-00368 to
A.L.K., grant 17-00-00366 to P.V.S.], Moscow University Development Program [grant PNR 5.13 to P.V.S.].

Author contributions: P.K. and A.P. assembled 705 ribosome complexes for the cryo-EM studies under the direct guidance of A.L.K.; E.B.P. and A.G.M. collected and processed cryo-EM data and built the final structural models; A.G.T. and D.I.S. performed competition binding assays under the direct guidance of A.A.B.; E.S.K. and I.A.O. performed in vitro inhibition assay; A.L.K., A.G.M., O.A.D., P.V.S., and A.A.B. supervised the experiments. All authors interpreted the results. E.B.P. and Y.S.P. prepared the figures. A.P., A.L.K., and Y.S.P. wrote the manuscript.

Received October 31, 2019; accepted December 27, 2019.

\section{REFERENCES}

Adams PD, Afonine PV, Bunkóczi G, Chen VB, Davis IW, Echols N, Headd JJ, Hung LW, Kapral GJ, Grosse-Kunstleve RW, et al. 2010. PHENIX: a comprehensive Python-based system for macromolecular structure solution. Acta Crystallogr D Biol Crystallogr 66: 213-221. doi:10.1107/S0907444909052925

Almutairi MM, Svetlov MS, Hansen DA, Khabibullina NF, Klepacki D, Kang HY, Sherman DH, Vázquez-Laslop N, Polikanov YS, Mankin AS. 2017. Co-produced natural ketolides methymycin and pikromycin inhibit bacterial growth by preventing synthesis of a limited number of proteins. Nucleic Acids Res 45: 95739582. doi:10.1093/nar/gkx673

Arenz S, Meydan S, Starosta AL, Berninghausen O, Beckmann R, Vázquez-Laslop N, Wilson DN. 2014. Drug sensing by the ribosome induces translational arrest via active site perturbation. Mol Cell 56: 446-452. doi:10.1016/j.molcel.2014.09.014

Arenz S, Bock LV, Graf M, Innis CA, Beckmann R, Grubmüller H, Vaiana AC, Wilson DN. 2016. A combined cryo-EM and molecular dynamics approach reveals the mechanism of ErmBLmediated translation arrest. Nat Commun 7: 12026. doi:10 .1038/ncomms12026

Brogden RN, Peters DH. 1994. Dirithromycin. A review of its antimicrobial activity, pharmacokinetic properties and therapeutic efficacy. Drugs 48: 599-616. doi:10.2165/00003495-199448040-00008

Bulkley D, Innis CA, Blaha G, Steitz TA. 2010. Revisiting the structures of several antibiotics bound to the bacterial ribosome. Proc Natl Acad Sci 107: 17158-17163. doi:10.1073/pnas.1008685107

Counter FT, Ensminger PW, Preston DA, Wu CY, Greene JM, FeltyDuckworth AM, Paschal JW, Kirst HA. 1991. Synthesis and antimicrobial evaluation of dirithromycin (AS-E 136; LY237216), a new macrolide antibiotic derived from erythromycin. Antimicrob Agents Chemother 35: 1116-1126. doi:10.1128/AAC.35.6.1116

Dinos GP, Connell SR, Nierhaus KH, Kalpaxis DL. 2003. Erythromycin, roxithromycin, and clarithromycin: use of slow-binding kinetics to compare their in vitro interaction with a bacterial ribosomal complex active in peptide bond formation. Mol Pharmacol 63: 617623. doi:10.1124/mol.63.3.617

Dunkle JA, Xiong L, Mankin AS, Cate JH. 2010. Structures of the Escherichia coli ribosome with antibiotics bound near the peptidyl transferase center explain spectra of drug action. Proc Natl Acad Sci 107: 17152-17157. doi:10.1073/pnas.1007988107

Emsley P, Cowtan K. 2004. Coot: model-building tools for molecular graphics. Acta Crystallogr D Biol Crystallogr 60: 2126-2132. doi:10.1107/S0907444904019158

Goddard TD, Huang CC, Meng EC, Pettersen EF, Couch GS, Morris JH, Ferrin TE. 2018. UCSF ChimeraX: meeting modern challenges in visualization and analysis. Protein Sci 27: 14-25. doi:10.1002/pro.3235 
Goldman RC, Zakula D, Flamm R, Beyer J, Capobianco J. 1994. Tight binding of clarithromycin, its 14-(R)-hydroxy metabolite, and erythromycin to Helicobacter pylori ribosomes. Antimicrob Agents Chemother 38: 1496-1500. doi:10.1128/AAC.38.7.1496

Grant T, Rohou A, Grigorieff N. 2018. cisTEM, user-friendly software for single-particle image processing. Elife 7: e35383. doi:10 $.7554 /$ eLife. 35383

Halfon Y, Matzov D, Eyal Z, Bashan A, Zimmerman E, Kjeldgaard J, Ingmer $\mathrm{H}$, Yonath $\mathrm{A}$. 2019. Exit tunnel modulation as resistance mechanism of $S$. aureus erythromycin resistant mutant. Sci Rep 9: 11460. doi:10.1038/s41598-019-48019-1

Hansen LH, Mauvais P, Douthwaite S. 1999. The macrolide-ketolide antibiotic binding site is formed by structures in domains II and $\mathrm{V}$ of $23 \mathrm{~S}$ ribosomal RNA. Mol Microbiol 31: 623-631. doi:10 $.1046 / j .1365-2958.1999 .01202 . x$

Kannan K, Kanabar P, Schryer D, Florin T, Oh E, Bahroos N, Tenson T, Weissman JS, Mankin AS. 2014. The general mode of translation inhibition by macrolide antibiotics. Proc Natl Acad Sci 111: 15958-15963. doi:10.1073/pnas. 1417334111

Khabibullina NF, Tereshchenkov AG, Komarova ES, Syroegin EA, Shiriaev DI, Paleskava A, Kartsev VG, Bogdanov AA, Konevega AL, Dontsova OA, et al. 2019. Structure of dirithromycin bound to the bacterial ribosome suggests new ways for rational improvement of macrolides. Antimicrob Agents Chemother 63: e02266-e02218. doi:10.1128/AAC.02266-18

Khatter H, Myasnikov AG, Natchiar SK, Klaholz BP. 2015. Structure of the human $80 \mathrm{~S}$ ribosome. Nature 520: 640-645. doi:10.1038/ nature14427

Konevega AL, Fischer N, Semenkov YP, Stark H, Wintermeyer W, Rodnina MV. 2007. Spontaneous reverse movement of mRNAbound tRNA through the ribosome. Nat Struct Mol Biol 14: 318324. doi:10.1038/nsmb1221

Milon P, Konevega AL, Peske F, Fabbretti A, Gualerzi CO, Rodnina MV. 2007. Transient kinetics, fluorescence, and FRET in studies of initiation of translation in bacteria. Methods Enzymol 430: 1-30. doi:10.1016/S0076-6879(07)30001-3

Noeske J, Wasserman MR, Terry DS, Altman RB, Blanchard SC, Cate JH. 2015. High-resolution structure of the Escherichia coli ribosome. Nat Struct Mol Biol 22: 336-341. doi:10.1038/nsmb .2994

Pettersen EF, Goddard TD, Huang CC, Couch GS, Greenblatt DM, Meng EC, Ferrin TE. 2004. UCSF Chimera: a visualization system for exploratory research and analysis. J Comput Chem 25: 16051612. doi:10.1002/jcc.20084

Polikanov YS, Osterman IA, Szal T, Tashlitsky VN, Serebryakova MV, Kusochek P, Bulkley D, Malanicheva IA, Efimenko TA, Efremenkova OV, et al. 2014a. Amicoumacin A inhibits translation by stabilizing mRNA interaction with the ribosome. Mol Cell 56: 531-540. doi:10.1016/j.molcel.2014.09.020

Polikanov YS, Steitz TA, Innis CA. 2014b. A proton wire to couple aminoacyl-tRNA accommodation and peptide-bond formation on the ribosome. Nat Struct Mol Biol 21: 787-793. doi:10.1038/nsmb .2871

Poulsen SM, Kofoed C, Vester B. 2000. Inhibition of the ribosomal peptidyl transferase reaction by the mycarose moiety of the antibiotics carbomycin, spiramycin and tylosin. J Mol Biol 304: 471-481. doi:10.1006/jmbi.2000.4229

Schlünzen F, Zarivach R, Harms J, Bashan A, Tocilj A, Albrecht R, Yonath A, Franceschi F. 2001. Structural basis for the interaction of antibiotics with the peptidyl transferase centre in eubacteria. Nature 413: 814-821. doi:10.1038/35101544

Schmeing TM, Huang KS, Strobel SA, Steitz TA. 2005. An induced-fit mechanism to promote peptide bond formation and exclude hydrolysis of peptidyl-tRNA. Nature 438: 520-524. doi:10.1038/ nature04152
Schüttelkopf AW, van Aalten DM. 2004. PRODRG: a tool for highthroughput crystallography of protein-ligand complexes. Acta Crystallogr D Biol Crystallogr 60: 1355-1363. doi:10.1107/ S0907444904011679

Sothiselvam S, Liu B, Han W, Ramu H, Klepacki D, Atkinson GC, Brauer A, Remm M, Tenson T, Schulten K, et al. 2014. Macrolide antibiotics allosterically predispose the ribosome for translation arrest. Proc Natl Acad Sci 111: 9804-9809. doi:10.1073/pnas .1403586111

Svetlov MS, Kommer A, Kolb VA, Spirin AS. 2006. Effective cotranslational folding of firefly luciferase without chaperones of the Hsp70 family. Protein Sci 15: 242-247. doi:10.1110/ps .051752506

Svetlov MS, Vázquez-Laslop N, Mankin AS. 2017. Kinetics of drug-ribosome interactions defines the cidality of macrolide antibiotics. Proc Natl Acad Sci 114: 13673-13678. doi:10.1073/pnas .1717168115

Svetlov MS, Plessa E, Chen CW, Bougas A, Krokidis MG, Dinos GP, Polikanov YS. 2019. High-resolution crystal structures of ribosome-bound chloramphenicol and erythromycin provide the ultimate basis for their competition. RNA 25: 600-606. doi:10 $.1261 /$ rna.069260.118

Tenson T, Lovmar M, Ehrenberg M. 2003. The mechanism of action of macrolides, lincosamides and streptogramin $B$ reveals the nascent peptide exit path in the ribosome. J Mol Biol 330: 1005-1014. doi:10.1016/S0022-2836(03)00662-4

Tereshchenkov AG, Shishkina AV, Karpenko WV, Chertkov VA, Konevega AL, Kasatsky PS, Bogdanov AA, Sumbatyan NV. 2016. New fluorescent macrolide derivatives for studying interactions of antibiotics and their analogs with the ribosomal exit tunnel. Biochemistry (Moscow) 81: 1163-1172. doi:10.1134/ S0006297916100138

Tereshchenkov AG, Dobosz-Bartoszek M, Osterman IA, Marks J, Sergeeva VA, Kasatsky P, Komarova ES, Stavrianidi AN, Rodin IA, Konevega AL, et al. 2018. Binding and action of amino acid analogs of chloramphenicol upon the bacterial ribosome. $J$ Mol Biol 430: 842-852. doi:10.1016/j.jmb.2018.01.016

Tu D, Blaha G, Moore PB, Steitz TA. 2005. Structures of MLSBK antibiotics bound to mutated large ribosomal subunits provide a structural explanation for resistance. Cell 121: 257-270. doi:10.1016/j cell.2005.02.005

Vazquez-Laslop N, Thum C, Mankin AS. 2008. Molecular mechanism of drug-dependent ribosome stalling. Mol Cell 30: 190-202. doi:10.1016/j.molcel.2008.02.026

Yan K, Hunt E, Berge J, May E, Copeland RA, Gontarek RR. 2005. Fluorescence polarization method to characterize macrolide-ribosome interactions. Antimicrob Agents Chemother 49: 3367-3372. doi:10.1128/AAC.49.8.3367-3372.2005

Ying L, Tang D. 2010. Recent advances in the medicinal chemistry of novel erythromycin-derivatized antibiotics. Curr Top Med Chem 10: 1441-1469. doi:10.2174/156802610792232042

Zhang K. 2016. Gctf: real-time CTF determination and correction. J Struct Biol 193: 1-12. doi:10.1016/j.jsb.2015.11.003

Zheng SQ, Palovcak E, Armache JP, Verba KA, Cheng Y, Agard DA. 2017. MotionCor2: anisotropic correction of beam-induced motion for improved cryo-electron microscopy. Nat Methods 14: 331-332. doi:10.1038/nmeth.4193

Zivanov J, Nakane T, Forsberg BO, Kimanius D, Hagen WJ, Lindahl E, Scheres SH. 2018. New tools for automated high-resolution cryoEM structure determination in RELION-3. Elife 7: e42166. doi:10 .7554/eLife. 42166

Zivanov J, Nakane T, Scheres SHW. 2019. A Bayesian approach to beam-induced motion correction in cryo-EM single-particle analysis. IUCrJ 6: 5-17. doi:10.1107/S205225251801463X 

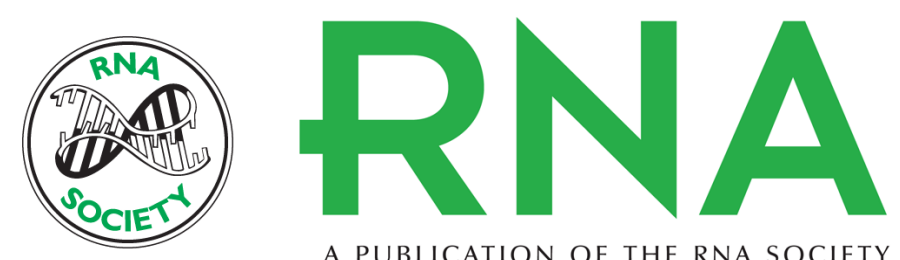

A PUBLICATION OF THE RNA SOCIETY

\section{Insights into the improved macrolide inhibitory activity from the high-resolution cryo-EM structure of dirithromycin bound to the $E$. coli 70 S ribosome}

Evgeny B. Pichkur, Alena Paleskava, Andrey G. Tereshchenkov, et al.

RNA 2020 26: 715-723 originally published online March 6, 2020

Access the most recent version at doi:10.1261/rna.073817.119

Supplemental Material

References

Creative Commons License

Email Alerting Service
http://rnajournal.cshlp.org/content/suppl/2020/03/06/rna.073817.119.DC1

This article cites 43 articles, 11 of which can be accessed free at: http://rnajournal.cshlp.org/content/26/6/715.full.html\#ref-list-1

This article is distributed exclusively by the RNA Society for the first 12 months after the full-issue publication date (see http://rnajournal.cshlp.org/site/misc/terms.xhtml). After 12 months, it is available under a Creative Commons License (Attribution-NonCommercial 4.0 International), as described at http://creativecommons.org/licenses/by-nc/4.0/.

Receive free email alerts when new articles cite this article - sign up in the box at the top right corner of the article or click here.

\section{|||||||| Providing Precise Solutions for} your research.

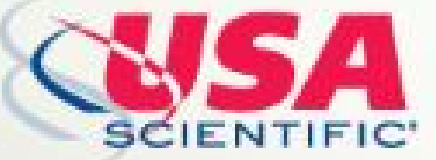

To subscribe to $R N A$ go to:

http://rnajournal.cshlp.org/subscriptions 